\title{
Comparative Evaluation of Ultrasonography and Computed Tomography in Pancreatic Lesions
}

\author{
SAMITA GUPTA, ${ }^{1}$ AMIT MITTAL, ${ }^{2}$ RAJ KUMAR ARION, ${ }^{3}$ RIKKI SINGAL ${ }^{4}$
}

\begin{abstract}
Background: Aims and Objectives - I) to do a comparative evaluation of ultrasonography and computed tomography in pancreatic lesions and assess their role as a useful diagnostic tool. 2) To correlate the USG and CT findings with fine needle aspiration cytology (FNAC)/ histopathological findings/ laboratory tests / therapeutic follow up wherever performed,

Material and Methods: The present study was carried out in the Department of Radio-diagnosis, Maharishi Markandeshwar Institute of Medical Sciences and Research, Mullana, Ambala. Thirty patients presenting with signs and symptoms of suspected pancreatic lesions referred from various wards and outpatient departments were included in the study.

Results: On USG, inflammatory lesions were diagnosed in 15 cases (50\%), and on CT scan, the diagnosis was made in 18 patients (60\%). Combining the USG \& CT findings of inflammatory lesions, the provisional radiological diagnosis of focal pancreatitis was made in I case but it was proven to be adenocarcinoma on FNAC. Provisional diagnosis of adenocarcinoma was made in 8 cases, lymphoma in 2 cases, macrocystic adenoma in I case and cystadenocarcinoma in I case on both USG and CT scan. However on FNAC, adenocarcinoma was proved in 10 patients, lymphoma was found in one case. Thus the provisional radiological diagnosis was correct in 28 patients (93.7\%).

Conclusion: Sonography detected pancreatic pathology in 27 cases (90\%) but CT scan detected pancreatic lesions in all the 30 patients (100\%). Present study had been undertaken to do a comparative evaluation of ultrasonography and computed tomography in pancreatic lesions and correlated the findings with fine needle aspiration cytology (FNAC) / HPE / laboratory tests / therapeutic follow up wherever performed. A need thus exists for multiple studies to produce significant in - road towards the appropriate imaging diagnosis of pancreatic pathologies as despite the rapid advances in imaging techniques, the overall impact of these modalities on the management and outcome of patients is still debatable.
\end{abstract}

Keywords: Pancreatitis; tumors; Ultrasonography; CT; Pathology.

\section{Introduction:}

Pancreatic lesions are a difficult diagnostic and therapeutic challenge owing to the deep seated location of pancreas. Acute or chronic pancreatitis may be associated with pancreatic calcification, pseudocysts, extrapancreatic phlegmons, hemorrhage and pancreatic necrosis/ abscess formation which can help the radiologist make an accurate

1. Assistant Prof. Department of Radiodiagnosis and Imaging, Maharishi Markandeshwer Institute of Medical Sciences and Research (MMIMSR), Mullana, Ambala, Haryana, India.

2. Professor and Head, Department of Radiodiagnosis and Imaging, MMIMSR, Mullana, Ambala, Haryana, India.

3. Professor, Department of Radiodiagnosis and Imaging, MMIMSR, Mullana, Ambala, Haryana, India.

4. Associate Prof. Department of Surgery, MMIMSR, Mullana, Ambala, Haryana, India.

Corresponding Author: Dr. Rikki Singal, Associate Prof. Department of Surgery, MMIMSR, Mullana, Ambala, Haryana, India. E mail - singalsurgery@yahoo.com, Mobile no 09996184795 , Fax No - 01731304550 diagnosis. ${ }^{1}$ Ultrasonography (USG) is a good modality because of its low cost, real time interactions, lack of bioeffects and wide availability. It can provide information about size, site and characteristics of pancreas, pancreatic lesion, diameter of the biliary and pancreatic ducts and site of obstruction. The presence or absence of lymph nodes or liver metastases can also be determined. More recently the use of echo enhanced Doppler sonography (Doppler after injection of contrast agent) has increased the sensitivity and specificity of this diagnostic modality. ${ }^{2}$

USG is operator dependent and has a limitation in obese patients and those with large amount of bowel gas. Computed tomography (CT) is a reliable modality and provides good definition of lesions and facilitates visualization of the entire extent of pancreatic pathology. Multiplanar threedimensional reconstruction techniques including volume rendering, maximum intensity projection and shaded surface display provide comprehensive information about the relationships and possible involvement of vascular structures 
and demonstrating local extension. ${ }^{3}$ But $\mathrm{CT}$ is expensive, exposes patient to ionizing radiation, may require long waiting periods for examination and may have difficulty in defining fat planes in lean patients. In addition USG-guided invasive manipulations of cystic pancreatic lesions /guided fine needle aspiration cytology (FNAC) of pancreatic masses are also easy to perform, quick and effective diagnostic methods but in some cases punctures and biopsies under $\mathrm{CT}$ control appear to be the imaging modality of choice. CT and USG are the most common utilized imaging modalities for evaluation of pancreatic pathologies. ${ }^{4}$ Hence USG should be used as screening procedure and CT should be used when USG is not helpful. Minniti S, et al performed a prospective study to compare sonography with helical CT in identification and staging of the ductal adenocarcinoma of the pancreas. The diagnostic imaging examinations were performed for presence of tumor, involvement of the peripancreatic vessels, any metastases in the liver and the resectability. Findings were compared with surgical findings. USG was more accurate in identification of adenocarcinoma than helical CT but CT was more accurate for resectability. The authors concluded that USG was more reliable than CT in identifying pancreatic adenocarcinoma but accuracy is similar in staging to CT. ${ }^{5}$ There is still a need to find a method which combines the accuracy of $\mathrm{CT}$ with similar availability and cost-effectiveness of USG.

\section{Materials and methods:}

The present study was carried out in the department of Radiodiagnosis, Maharishi Markandeshwar Institute of Medical Sciences and Research, Mullana, Ambala. Thirty patients presenting with signs and symptoms of suspected pancreatic lesions referred from various wards and outpatient departments were included in the study. A complete history of patients was taken and detailed clinical examination performed after obtaining the written informed consent in all the cases. Relevant laboratory investigations as mentioned in the proforma were done. Following this patients were subjected to sonography followed by computed tomography examination. All findings were recorded as per proforma attached.

\section{Equipment:}

Ultrasonography (USG)

- Machine - Logiq 500 Pro (GE)

- With a $2.5-3.5 \mathrm{MHz}$ convex transducer

Computed tomography (CT)

- Machine - Somatom Emotion Scanner (Siemens) - single slice spiral CT scanner
Sonography - Overnight fasting was preferred. The patient was made to lie down on the couch. A coupling agent was applied liberally to patient's skin to act as acoustic window removing the air between transducer and patient's skin surface and allow swift movement of the transducer. A general abdominal survey was done with a $3.5 \mathrm{MHz}$ convex transducer with transverse, longitudinal and other desired planes. Pancreas was visualized by transverse scans in midline below the xiphoid process using the related vascular landmarks to identify it. The probe was made oblique to visualize the gland in its entirety. Using left kidney as an acoustic window, the tail of pancreas was visualized anterior to its upper pole. Wherever required, pancreatic and left upper quadrant visibility was improved by having patient drink a glass of water or making the patient stand erect. Color Doppler was done for vascularity of pancreas, pancreatic lesion and associated vascular structures. Various sonographic findings regarding size, shape, contour, echotexture of pancreas and pancreatic lesion, peripancreatic area were noted. Other associated findings like gall bladder stones, status of liver, ascites, pleural effusion, lymph nodes, calcification, vascular involvement etc were also searched. Findings were recorded as per proforma.

Following USG patient were subjected to CT examination.

Computed tomography (CT) - Patients were instructed to report after fasting for at least six hours. All patients were given oral contrast required for the opacification of duodenum and the bowel. Patients were positioned supine and scout image of abdomen taken. The region of interest was defined and extended from the domes till lower poles of kidneys. Plain sections were taken of the pancreatic area. Contrast enhanced CT scan of abdomen was then obtained after intravenous administration of $80-120 \mathrm{ml}$ of non-ionic contrast medium (Iohexol) containing $300 \mathrm{mg} / \mathrm{ml}$ of iodine. The amount of contrast was varied according to the patient's body weight, clinical and renal status. Lateral decubitus position was used in some cases. Scan slices were taken as $10 \mathrm{~mm}$ contiguous sections (Pitch - 1.5) over the entire upper abdomen and the volumetric data acquired reconstructed with $3 \mathrm{~mm}$ thin slices. Multiplanar reconstructions were done wherever required. Axial sections were studied in detail regarding size, shape, density, enhancing pattern of pancreas and pancreatic lesion, peripancreatic infiltration/fat planes, any vascular complications etc. Other structures were also looked for any pathology particularly liver and biliary tree. Associated findings like ascites, pleural effusion, and lymphadenopathy were also noted. The findings were recorded as per proforma attached. The results of sonography and computed tomography were evaluated in each case and 
findings correlated with fine needle aspiration cytology (FNAC) / histopathological findings/ laboratory tests / therapeutic follow up wherever performed.

\section{Observations and results:}

In the present series, age of the patients ranged from 11-80 years. Maximum number of patients was in the age range of 41-50 years followed by 31-40years. The mean age of occurrence of pancreatitis was around 45 years and malignant pancreatic lesions were around 62 years. Male patients outnumbered the female patients in this study. There were 18 males $(60 \%)$ and 12 females $(40 \%)$. Maximum no of males (6) and females (3) were in the age range of 41-50

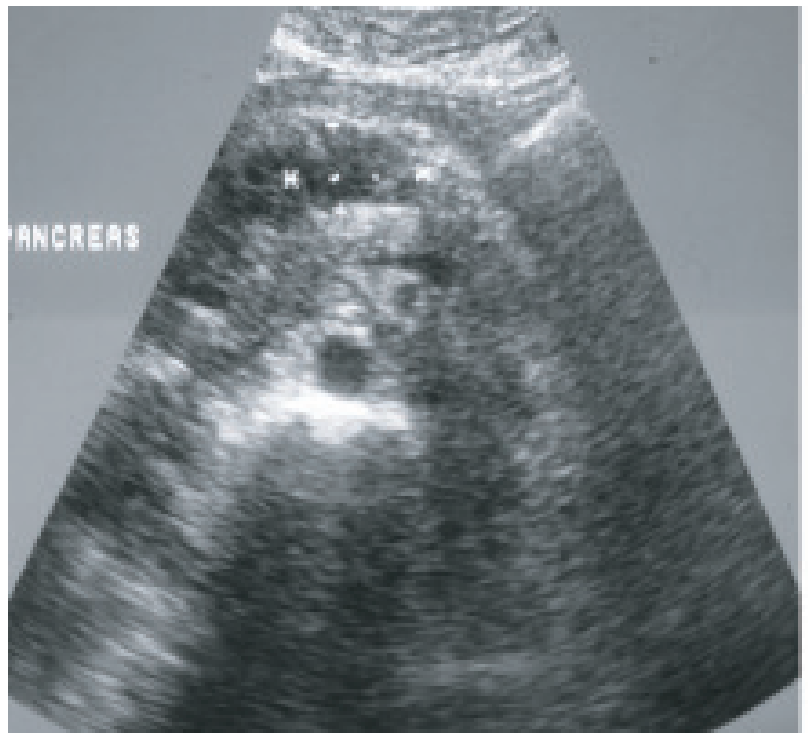

Fig.-1a: USG -focal hypoechoic lesion is seen in body of pancreas

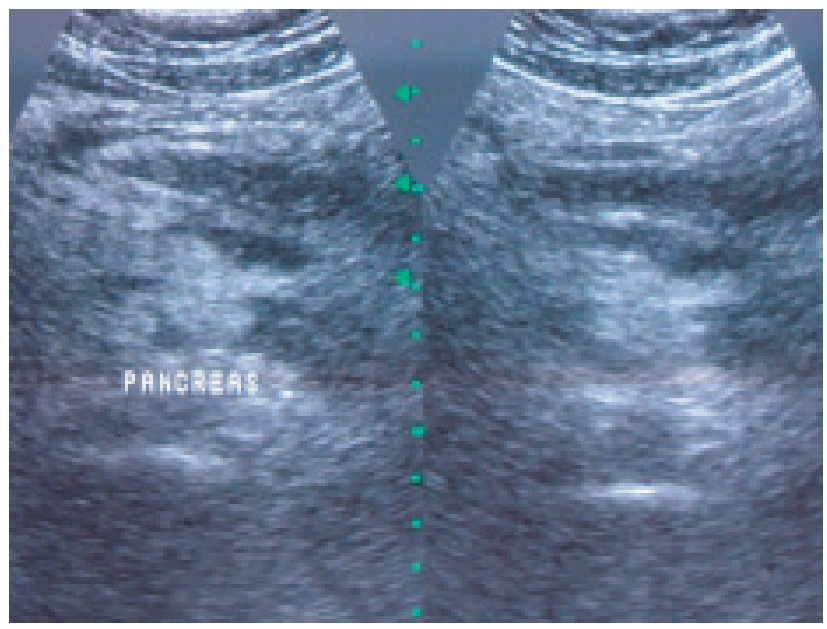

Fig.-2a: USG shows peripancreatic collection with necrosis years. Most common symptom was pain abdomen seen in 22 patients. The pain was present in the epigastric region radiating to back. Next common symptom was nausea and vomiting in 20 patients. Weight loss was seen in 16 patients and jaundice was seen in 10 patients. Many of the patients presented with more than one symptom. However lump in the abdomen was detected in 4 cases only. Serum amylase was done in 20 patients. A raised level of $>140 \mathrm{IU} / \mathrm{L}$ was found in 8 patients of acute pancreatitis and a normal level between 35-140 IU/L was seen in 12 patients. Serum lipase was found to be more sensitive being positive in 10 cases (Table-1).

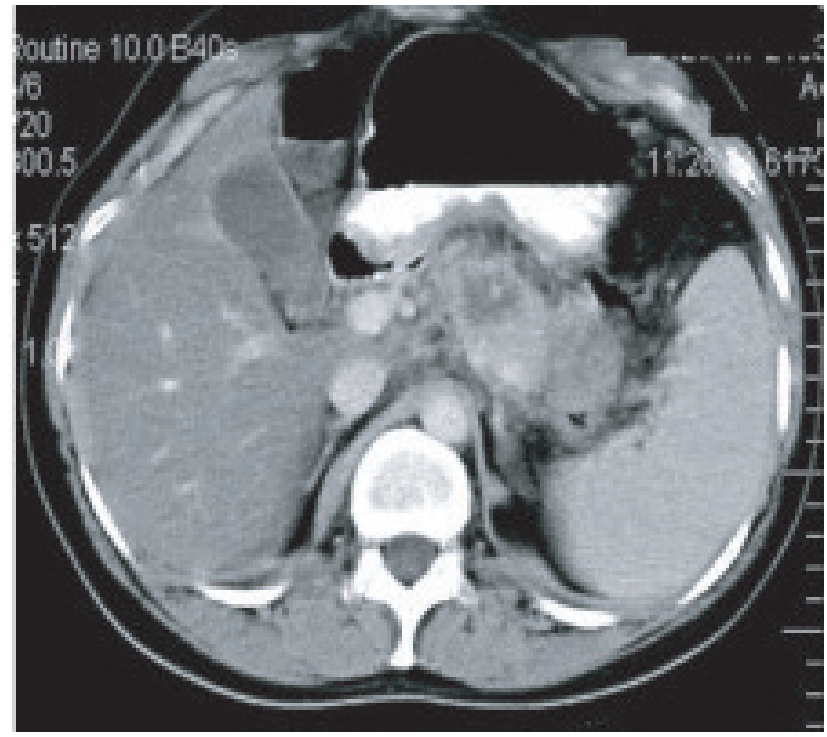

Fig.-1b: CECT-Focal ill defined non enhancing hypodense area in body

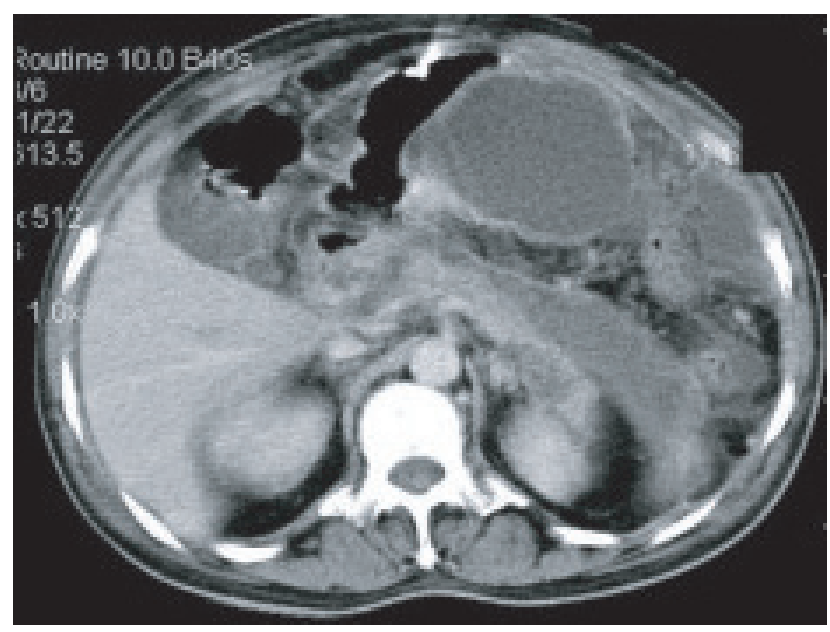

Fig.-2b: CT showed acute necrotizing pancreatitis with peripancreatic collections 


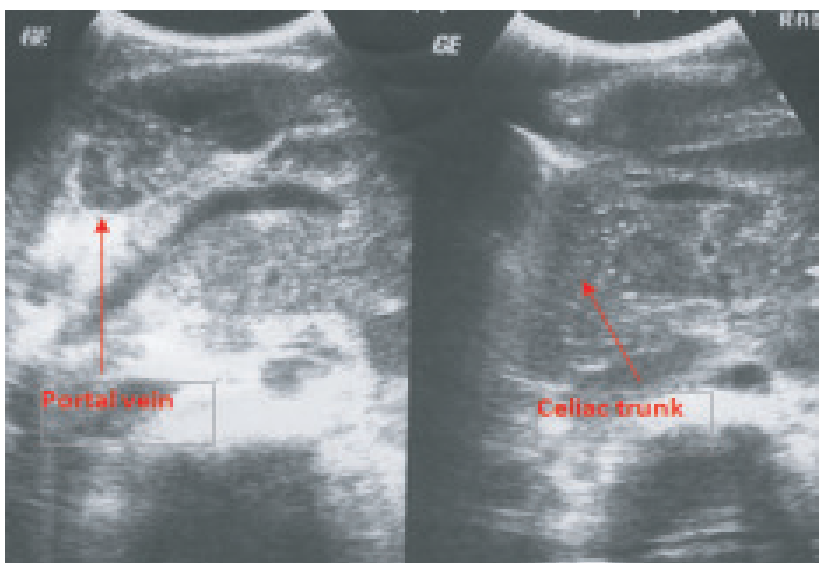

Fig.3a: USG showed a large hypoechoic mass lesion involving pancreas with encasement of celiac trunk and portal vein.

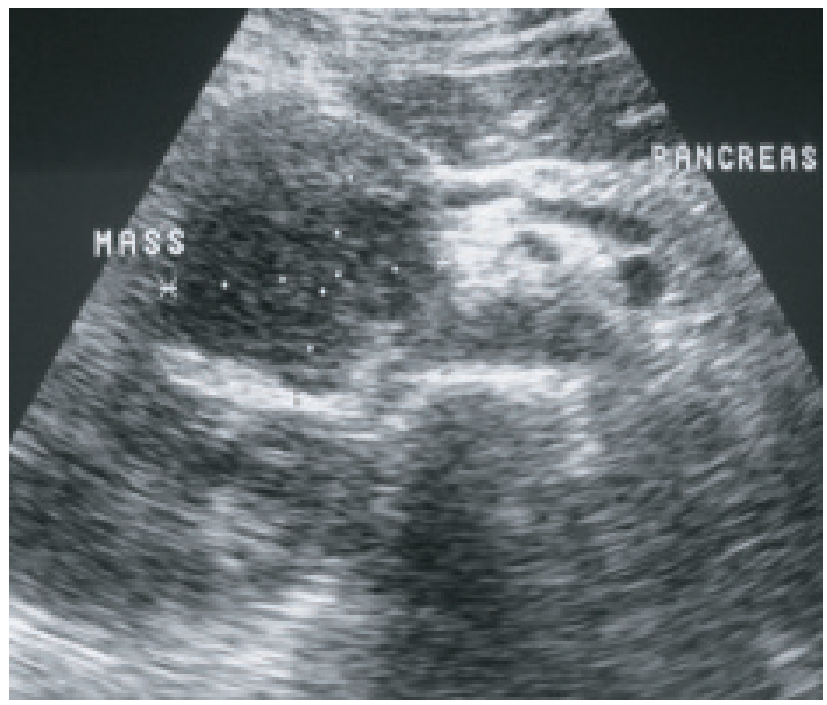

Fig.-4a: USG - hypoechoic mass seen in head region

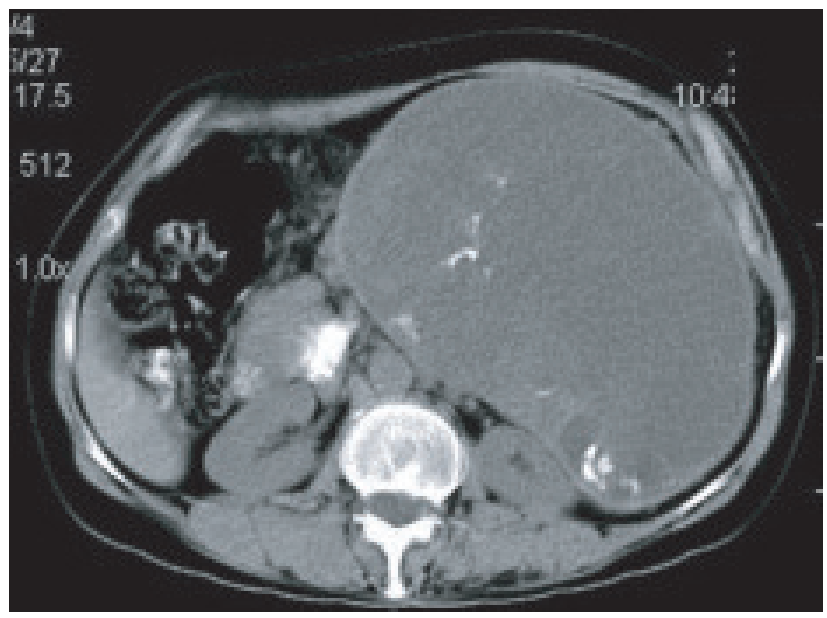

Fig.-5a: Plain CT scan-large cystic mass with central and peripheral calcification

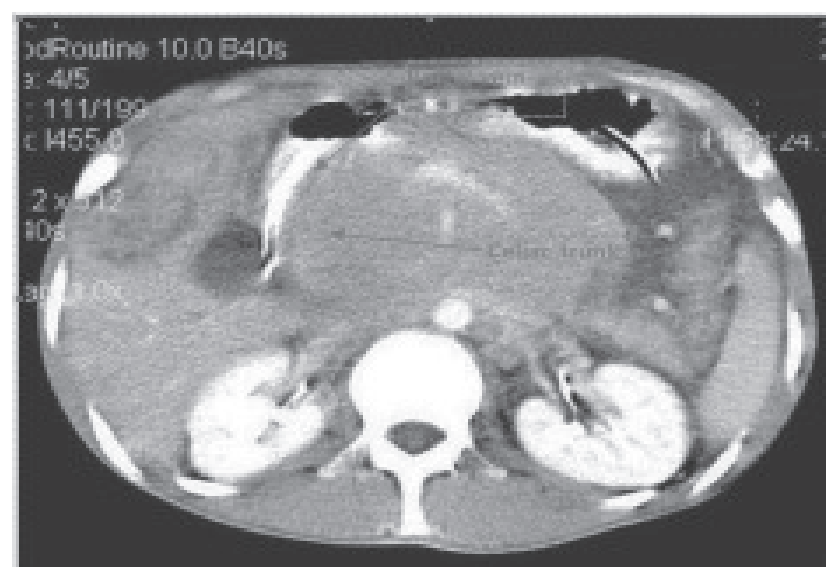

Fig.-3b: CECT - large moderately enhancing mass lesion with diffuse involvement of pancreas with encasement of celiac trunk and portal vein with focal lesion in liver

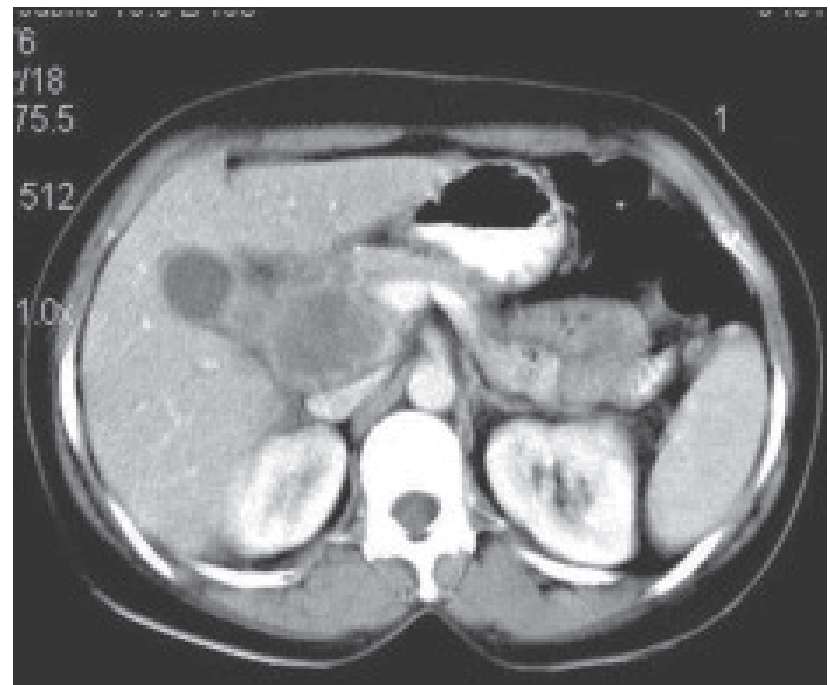

Fig.-4b: CECT- hypodense mass with heterogenous enhancement with compression of $2^{\text {nd }}$ part of duodenum.

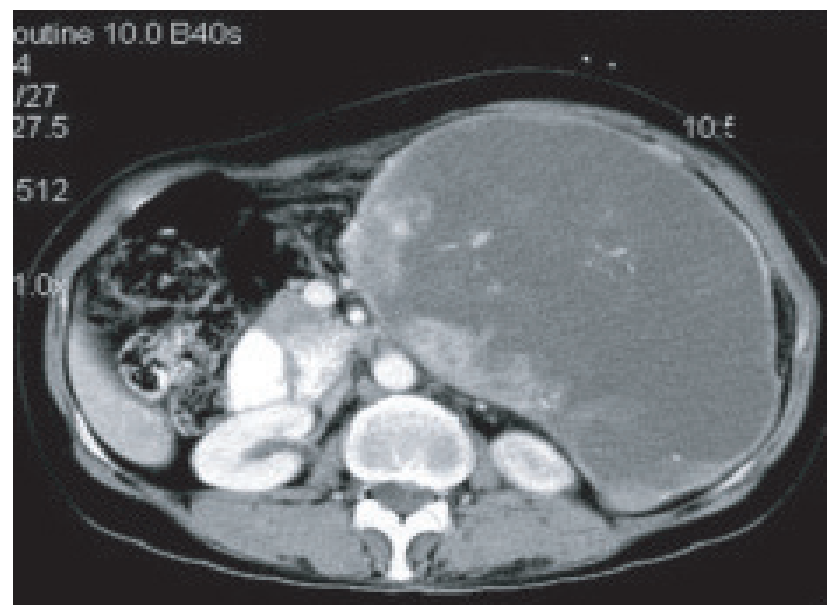

Fig.-5b: Post contrast CT scan heterogenous enhancement seen with enhancing solid components 


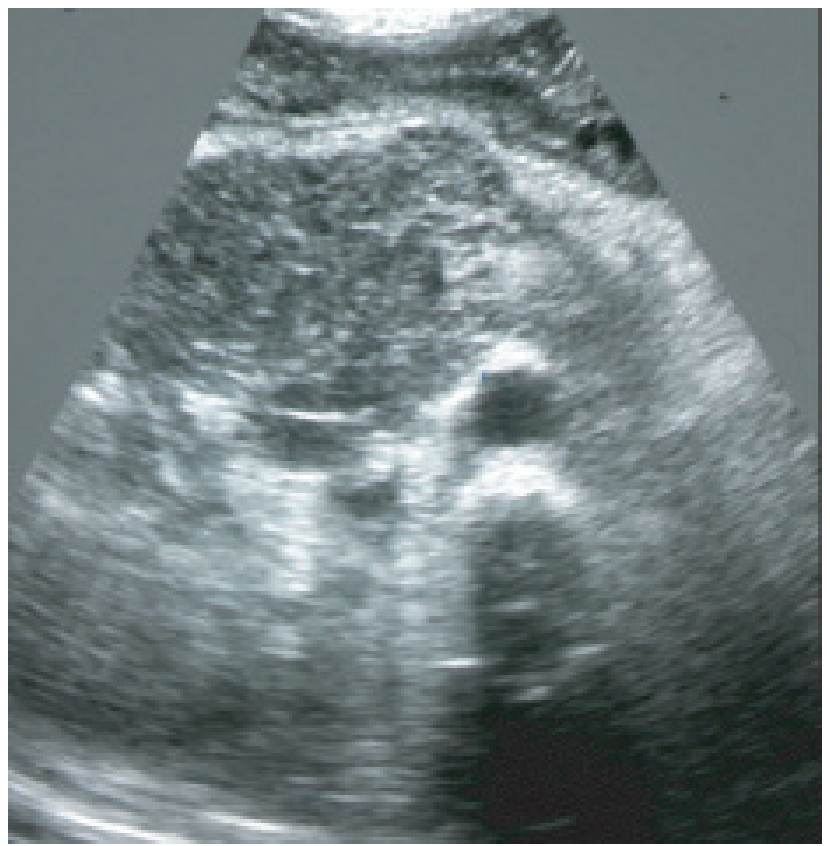

Fig.-6a: USG large hypoechoic mass head

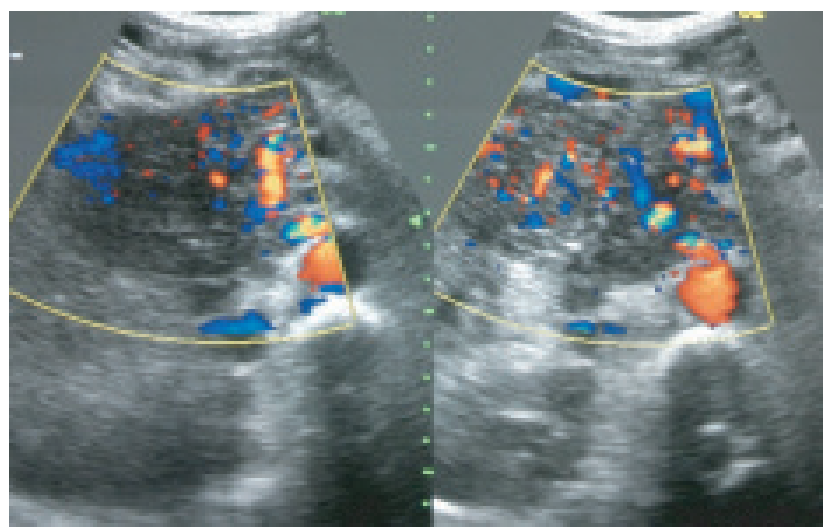

Fig.-6b: Increased vascularity on doppler

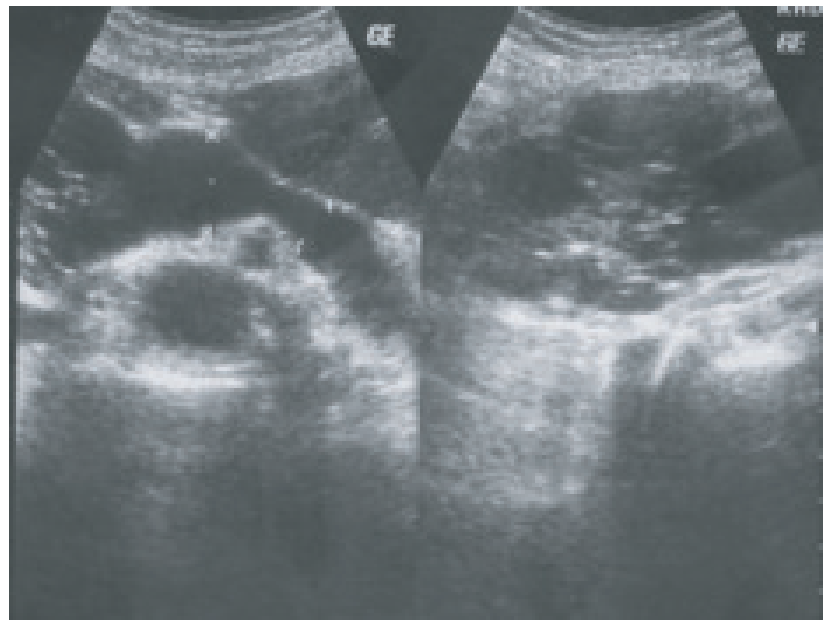

Fig.-7a: USG - showed multicystic mass in head region communicating with PD. No calcification seen.

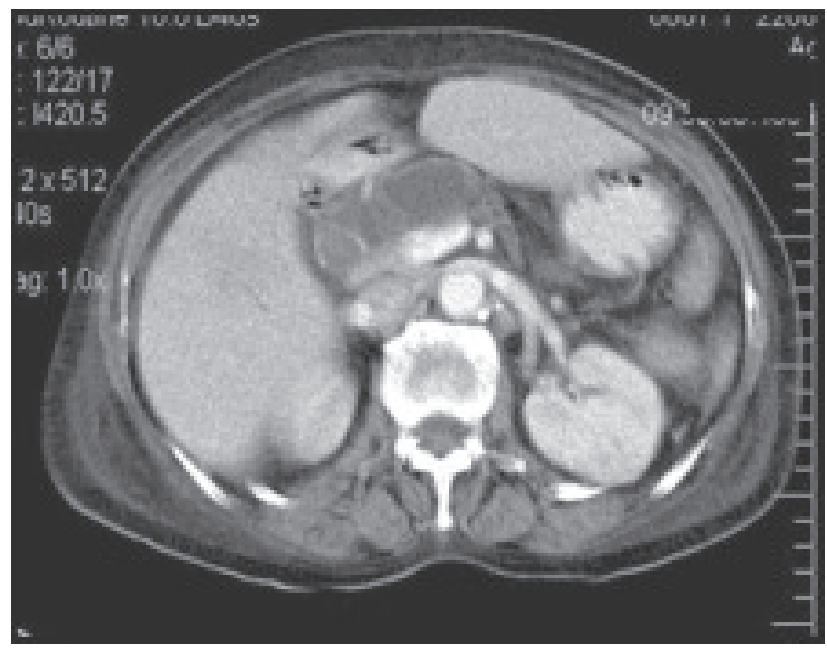

Fig.-7b: CT- showed mass communicating with pancreatic duct

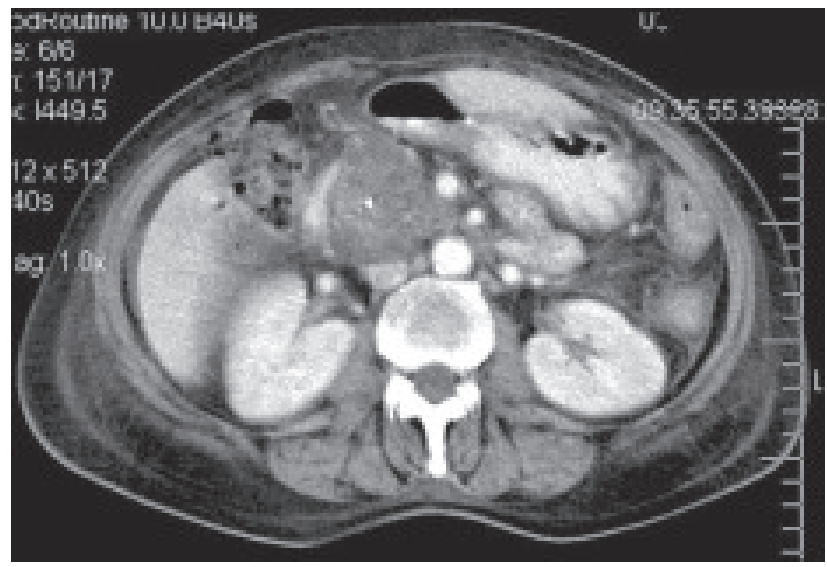

Fig.-7c: CECT - showed multicystic mass in head region with heterogenous enhancement with speck of calcification

Table-1

Serum amylase/lipase values $(n=30)$

\begin{tabular}{lcccc}
\hline Serum Amylase & \multicolumn{2}{c}{ Amylase } & \multicolumn{2}{c}{ Lipase } \\
/Lipase & No. of cases & $\%$ age & No. of cases & $\%$ Age \\
\hline Raised & 8 & $26.6 \%$ & 12 & $40 \%$ \\
Normal & 12 & $40 \%$ & 10 & $33.3 \%$ \\
Not Done & 10 & $33.3 \%$ & 8 & $26.6 \%$ \\
\hline Total & 30 & $100 \%$ & 30 & $100 \%$ \\
\hline
\end{tabular}

\section{Table-II}

Presence of pancreatic lesion on sonography $(n=30)$

\begin{tabular}{lcc}
\hline Presence of lesion & No. of patients & $\%$ Age \\
\hline Lesion detected & 27 & $90 \%$ \\
Obscured pancreas due to bowel gases & 3 & $10 \%$ \\
\hline Total & 30 & $100 \%$ \\
\hline
\end{tabular}


Sonographic observations - Ultrasound could detect pancreatic lesion in 27 out of 30 patients $(90 \%)$. In 3 patients pancreas could not be visualised as it was obscured by overlying bowel gases (in 2 cases the entire pancreas was not visualised and in one case the tail region was not visualised) (Table-2). Hence in these 3 cases, pancreatic lesions could not be detected on USG; however they were seen on CT. Size of the pancreas was enlarged in 22 patients $(73.3 \%)$ on sonography. The enlargement was either due to inflammatory changes /malignant pathology. Pancreas was atrophic in 5 cases of chronic pancreatitis. Pancreas was not visualised in remaining 3 cases because of overlying bowel gases. Involvement of pancreas was diffuse in 16 patients comprising $53.3 \%$ of total cases. 11 patients showed focal involvement of pancreas involving head, body or tail. Majority of the lesions were seen in the region of head. The contour of pancreas was regular in 8 cases $(26.6 \%)$ whereas it was irregular in 19 cases $(63.3 \%)$. Pancreas was not visualised in 3 cases because of overlying bowel gases.
Majority of the lesions - 16 cases (53.3\%) were heterogeneously hypoechoic in echotexture with areas of calcification/necrosis within them. The lesions were homogenously hypoechoic in 10 cases $(33.3 \%)$. Echotexture of pancreas was normal in one case (Table-3). Calcification was seen in 9 pancreatic lesions on sonography comprising $30 \%$ of cases. Rest of the cases showed no evidence of any calcification. Pancreatic duct more than $3 \mathrm{~mm}$ in calibre was considered dilated for any age. Pancreatic duct dilatation was seen in 10 patients; however in 17 patients it was normal in caliber (Table-4).

Intrapancreatic fluid collections were seen in 4 patients. Peripancreatic fluid collections were seen in 8 patients; predominantly in the region of lesser sac. Pseudocysts were seen in 3 patients of chronic pancreatitis. There was no evidence of any fluid collection in 15 patients including 3 patients in whom pancreas was not visualised (Table-5). Gall stones were seen in 8 patients of pancreatitis. CBD stone was seen in 1 case. Ascites was seen in 7 cases. Pleural

Table -III

Echotexture of pancreatic lesions $(n=30)$

\begin{tabular}{|c|c|c|c|c|c|}
\hline \multicolumn{3}{|c|}{ USG Findings } & \multicolumn{3}{|c|}{ CT Scan Findings } \\
\hline Echogenicity & Number of cases & $\%$ Age & Density & Number of cases & $\%$ AGE \\
\hline Normal & 1 & $3.3 \%$ & Hypodense & 13 & $43.3 \%$ \\
\hline Hypoechoic & 10 & $33.3 \%$ & Heterogeneous & 14 & $46.6 \%$ \\
\hline Heterogeneous & 16 & $53.3 \%$ & Cystic component & 2 & $6.6 \%$ \\
\hline Not Visualised & 3 & $10 \%$ & Pseudocysts & 3 & $10 \%$ \\
\hline Total & 30 & $100 \%$ & Total & 30 & $100 \%$ \\
\hline
\end{tabular}

Table - IV

Pancreatic duct dilatation on sonography $(n=30)$

\begin{tabular}{lcclcr}
\hline & USG & & \multicolumn{2}{c}{ CT } \\
Pancreatic duct & Number of cases & \%Age & Pancreatic duct & Number of cases & \%Age \\
\hline Dilated & 10 & $33.3 \%$ & Dilated & 10 & $33.3 \%$ \\
Not Dilated & 17 & $56.6 \%$ & Not Dilated & 20 & $66.6 \%$ \\
Not Visualised & 3 & 10 & Not Visualised & 30 & $100 \%$ \\
\hline Total & 30 & $100 \%$ & Total
\end{tabular}

Table -V

Fluid collections on sonography $(n=30)$

\begin{tabular}{|c|c|c|c|c|c|}
\hline \multicolumn{3}{|c|}{ USG } & \multicolumn{3}{|c|}{$\mathrm{CT}$} \\
\hline Fluid collections & Number of cases & $\%$ Age & Fluid collections & Number of cases & $\%$ Age \\
\hline Intrapancreatic & 4 & $13.3 \%$ & Intrapancreatic & 5 & $16.6 \%$ \\
\hline Peripancreatic & 8 & $26.6 \%$ & Peripancreatic & 11 & $36.6 \%$ \\
\hline Pseudocyst & 3 & $10 \%$ & Pseudocyst & 0 & \\
\hline No fluid collection & 15 & $50 \%$ & No fluid collection & 14 & $46.6 \%$ \\
\hline Total & 30 & $100 \%$ & Total & 30 & $100 \%$ \\
\hline
\end{tabular}


effusion was seen in 6 cases which was left sided in 5 cases and right sided in 1 case. Enlarged lymph nodes were seen in 6 cases. Extrahepatic and intrahepatic biliary dilatation was seen in 8 cases. Hepatic metastases were seen in 6 cases of malignant pancreatic lesions (Table-6). Ultrasound could not detect pancreas in 3 cases because of overlying bowel gases. Out of 15 cases of inflammatory pathologies, acute pancreatitis was seen in 7 cases, acute exacerbation of chronic pancreatitis was seen in 2 cases. Chronic pancreatitis was seen in 5 cases which included 3 cases of pseudocyst formation and 2 cases without pseudocyst formation. Focal acute pancreatitis in the region of pancreatic body was seen in 1 case. Provisional diagnosis of malignant lesions was made in 12 cases on sonography which included adenocarcinoma in 8 cases, lymphoma in 2 cases, macrocystic adenoma in 1 case and cystadenocarcinoma in 1 case (Table-7).

Observations on computed tomography (CT scan) Pancreatic lesions were seen in all thirty patients on CT scan. 3 cases which were not visualised on sonography were also seen on CT. Pancreas was enlarged in 24 cases $(80 \%)$ out of

Table-VI

Additional sonographic and CT findings

\begin{tabular}{lcclcc}
\hline & USG & & \multicolumn{2}{c}{ CT } \\
Findings & Number of cases & \%Age & Findings & Number of cases & $\%$ Age \\
\hline Gall Bladder Stones & 8 & $26.6 \%$ & Gall Bladder Stones & 6 & $20 \%$ \\
CBD stone & 1 & $3.3 \%$ & CBD stone & 1 & $3.3 \%$ \\
IHBR/CBD Dilatation & 9 & $30 \%$ & IHBR/CBD Dilatation & 9 & $30 \%$ \\
Ascites & 7 & $23.3 \%$ & Ascites & 7 & $23.3 \%$ \\
Lymphadenopathy & 6 & $20 \%$ & Lymphadenopathy & 9 & $30 \%$ \\
Pleural effusion & 6 & $20 \%$ & Pleural effusion & 6 & $20 \%$ \\
Liver Metastasis & 6 & $20 \%$ & Liver Metastasis & 6 & $20 \%$ \\
Invasion/involvement of & 0 & & Invasion/involvement of & 4 & $13.3 \%$ \\
adjacent structures & & & adjacent structures & & \\
Vascular involvement & 0 & & Vascular involvement & 7 & $23.3 \%$ \\
\hline
\end{tabular}

Table-VII

Provisional sonographic and CT diagnosis

\begin{tabular}{lcclcc}
\hline Diagnosis & Number of cases & \%Age & Diagnosis & Number of cases & \%Age \\
\hline Inflammatory & 15 & $50 \%$ & Inflammatory & 18 & $60 \%$ \\
Acute pancreatitis & 7 & $23.3 \%$ & Acute edematous pancreatitis & 4 & $13.3 \%$ \\
& & & Acute necrotizing pancreatitis & 5 & $16.6 \%$ \\
Acute on chronic pancreatitis & 2 & $6.6 \%$ & Acute on chronic pancreatitis & 2 & $6.6 \%$ \\
Chronic pancreatitis with & 3 & $10 \%$ & Chronic pancreatitis with & 3 & $10 \%$ \\
pseudocyst & & & pseudocyst & \\
Chronic pancreatitis without & 2 & $6.6 \%$ & Chronic pancreatitis without & 2 & $6.6 \%$ \\
pseudocyst & 1 & & pseudocyst & 2 & $6.6 \%$ \\
Focal pancreatitis & 3 & $10 \%$ & Pancreas not visualised & 0 & $0 \%$ \\
Pancreas not visualised & 12 & $40 \%$ & Malignant & 12 & $40 \%$ \\
Malignant & 8 & $26.6 \%$ & Adenocarcinoma & 8 & $26.6 \%$ \\
Adenocarcinoma & 2 & $6.6 \%$ & Lymphoma & 2 & $6.6 \%$ \\
Lymphoma & 1 & $3.3 \%$ & Macrocystic adenoma & 1 & $3.3 \%$ \\
Macrocystic adenoma & 1 & $3.3 \%$ & Cystadenocarcinoma & 1 & $3.3 \%$ \\
Cystadenocarcinoma & 30 & $100 \%$ & & & \\
Total & & & & \\
\hline
\end{tabular}


total 30 cases. Enlargement was either due to inflammatory/ malignant pathology. These 24 cases also included three cases which were not visualised on sonography. CT scan showed focal enlargement in region of pancreatic tail in 1 case and diffuse enlargement of pancreas in another 2 cases. Pancreas was atrophic in 5 cases of chronic pancreatitis. Size of pancreas was normal in one case. Diffuse involvement of pancreas was seen in 18 cases $(60 \%)$ and focal involvement in 12 cases (40\%) predominantly in the region of head - 9 cases ( $30 \%$ ), followed by body -2 cases $(6.6 \%)$ and least common in the region of tail - only 1 case $(3.3 \%)$. Out of 3 cases which were not seen on sonography 2 cases showed diffuse involvement and 1 case showed focal involvement in region of pancreatic tail on CT scan. The margins of pancreas showed well defined outline in 10 cases whereas the outlines were ill defined in 20 cases.

The lesions seen on CT were either homogenously or heterogeneously hypodense. ${ }^{13}$ cases were hypodense comprising $43.3 \%$ of cases. 14 cases were heterogeneously hypodense comprising $46.6 \%$ of cases. Out of these 14 heterogeneous lesions, 2 cases showed predominantly cystic component. Pseudocysts were seen in 3 cases (Table-3). Calcification was seen in pancreatic lesions in 11 cases on CT (36.6\%) and was absent in 19 cases. CT was more sensitive than USG in detecting calcification in two cases. Out of total of 30 cases pancreatic duct dilatation was seen in 10 cases $(33.3 \%)$. The calibre of pancreatic duct more than $3 \mathrm{~mm}$ was considered dilated for any age. Main pancreatic duct (MPD) was normal in 20 cases $(66.6 \%)$ (Table-4).
Maximum number of patients showed heterogeneous moderate enhancement on post contrast CT scans i.e. 18 cases $(60 \%)$. This was followed by homogenous moderate enhancement in 7 cases $(23.3 \%)$. Few of the pancreatic lesions i.e. 5 cases $(16.6 \%)$ including chronic pancreatitis showed no enhancement on CECT. The necrotic or non enhancing areas were evaluated after contrast enhanced CT scan. Maximum number of patients showed no evidence of necrosis i.e. in 15 cases $(50 \%)$. There was presence of necrosis in almost equal proportion in range of $30-50 \%$ and $>50 \%$ in 4 cases of acute pancreatitis. 10 cases of malignant pathologies showed e/o necrotic areas.

Intrapancreatic fluid collections were seen only in 5 patients. Peripancreatic fluid collections including pseudocysts were seen in 11 patients predominantly in the region of lesser sac. Some of the patients showed both intra and peripancreatic fluid collections. There was no e/o any fluid collection in 15 patients (Table-5). Peripancreatic stranding and fascial plane thickening was seen in 12 patients $(40 \%)$. It was seen predominantly in inflammatory lesions, while 2 cases of malignant lesions also showed similar findings due to local involvement. No stranding/fascial thickening was seen in 18 cases $(60 \%)$. Gall stones were missed in 2 patients out of total 8 patients in whom USG was able to detect cholelithiasis.CBD stone was seen in 1 case. Enlarged regional and distant lymph nodes were seen in 9 cases while USG showed lymphadenopathy in 6 patients. Out of 12 cases of malignant lesions - 4 patients showing e/o local extension/ invasion. There was evidence of metastases to liver in 6 patients. Involvements of the peripancreatic major vessels portal vein/IVC/SMV/SMA were seen in 7 patients including

Table - VIII

Comparative evaluation of provisional USG and CT diagnosis

\begin{tabular}{lccccc}
\hline Provisional diagnosis & \multicolumn{2}{c}{ USG } & & \multicolumn{2}{c}{ CT } \\
\cline { 2 - 3 } \cline { 5 - 6 } & Number of Cases & $\%$ Age & & Number of Cases & $\%$ age \\
\hline Inflammatory & 15 & $50 \%$ & 18 & $60 \%$ \\
Acute pancreatitis & 7 & $23.3 \%$ & & $30 \%$ \\
Acute on chronic pancreatitis & 2 & $6.6 \%$ & 2 & $6.6 \%$ \\
Chronic pancreatitis with pseudocyst & 3 & $10 \%$ & 3 & $10 \%$ \\
Chronic pancreatitis without pseudocyst & 2 & $6.6 \%$ & 2 & $6.6 \%$ \\
Focal pancreatitis & 1 & $3.3 \%$ & & 2 & $6.6 \%$ \\
MALIGNANT & 12 & $40 \%$ & 12 & $40 \%$ \\
Pancreas Not Visualised & 3 & $10 \%$ & 0 & $0 \%$ \\
\hline Total & 30 & $100 \%$ & 30 & $100 \%$ \\
\hline
\end{tabular}


Table - IX

Comparative evaluation of radiological diagnosis with final diagnosis by FNAC/HPE/lab tests/follow up

\begin{tabular}{lcclcc}
\hline Radiological diagnosis & Number of cases & $\%$ Age & Final diagnosis & Number of cases & Age \\
\hline Acute edematous pancreatitis & 4 & $13.3 \%$ & Acute edematous pancreatitis & 4 & $13.3 \%$ \\
Acute necrotising pancreatitis & 5 & $16.6 \%$ & Acute necrotising pancreatitis & 5 & $16.6 \%$ \\
Acute on chronic pancreatitis & 2 & $6.6 \%$ & Acute on chronic pancreatitis & 2 & $6.6 \%$ \\
Chronic pancreatitis with pseudocyst & 3 & $10 \%$ & Chronic pancreatitis with pseudocyst & 3 & $10 \%$ \\
Chronic pancreatitis without pseudocyst & 2 & $6.6 \%$ & Chronic pancreatitis without pseudocyst 2 & $6.6 \%$ \\
Focal Pancreatitis1. & & & & & \\
1 Body & 1 & $3.3 \%$ & 1. Adenocarcinoma & 1 & $3.3 \%$ \\
2 Tail & 1 & $3.3 \%$ & 2 . Focal pancreatitis & 1 & $3.3 \%$ \\
Adenocarcinoma & 8 & $26.6 \%$ & Adenocarcinoma & 9 & $30 \%$ \\
Lymphoma & 2 & $6.6 \%$ & Lymphoma & 1 & $3.3 \%$ \\
Macrocystic adenoma & 1 & $3.3 \%$ & Macrocystic adenoma & 1 & $3.3 \%$ \\
Cystadenocarcinoma & 1 & $3.3 \%$ & Cystadenocarcinoma & 16 & $53 \%$ \\
Inflammatory & 18 & $60 \%$ & Inflammatory & 14 & $46.6 \%$ \\
Malignant & 12 & $40 \%$ & Malignant & 30 & $100 \%$ \\
\hline Total & 30 & $100 \%$ & Total & &
\end{tabular}

portal vein thrombus, encasement of PV/SMV/SMA and thrombosis of SMA /SMV and splenic vein. Features of obstructive biliopathy in form of extra/intrahepatic biliary dilatation were noted in 9 patients. Ascites and pleural effusion was seen in 7 and 6 cases respectively (Table-VI).

In accordance with the imaging findings on $\mathrm{CT}$, provisional diagnosis was made in all the 30 patients. The pancreatic lesions detected on $\mathrm{CT}$ were characterized into inflammatory and malignant lesions. 18 patients had inflammatory pathology including acute, acute on chronic, chronic and focal pancreatitis. 12 patients had malignant pathology including adenocarcinoma, lymphoma, macrocystic adenoma and cystadenocarcinoma (Table-VII). Pancreatic lesions were seen in all thirty patients on CT scan. 3 cases which were not visualised on sonography due to overlying bowel gases were also seen on CT. These included 1 case of acute edematous pancreatitis, 1 case of acute necrotising pancreatitis and another case of focal acute pancreatitis in tail region. 12 malignant pancreatic lesions were detected by USG and CT both, though the radiological diagnosis was incorrect in 2 cases. Provisional radiological diagnosis was made in 27 out of 30 patients by USG including 3 cases in which pancreas was not visualised due to overlying bowel gases, however CT could detect pancreatic lesions in all 30 patients (Table-VIII). 1 case which was diagnosed radiologically as focal pancreatitis in body region on both
CT and USG was proven to be adenocarcinoma on HPE. Another case in which radiological diagnosis proved wrong was lymphoma which was proven to be adenocarcinoma on FNAC. Thus USG was non-diagnostic in 5 cases $(90 \%$ sensitive and $83.6 \%$ specific) and CT in 2 cases $(100 \%$ sensitive and $93.4 \%$ specific) on correlation with final diagnosis (Table-IX).

\section{Discussion:}

The early clinical diagnosis of pancreatic lesions is fraught with difficulty. Unfortunately, the initial symptoms are often quite non-specific and subtle in onset. A number of continually evolving imaging modalities is available to diagnose the pancreatic pathologies. These include plain Xrays, transabdominal ultrasonography (TAS), computed tomography (CT), magnetic resonance imaging (MRI), endoscopic retrograde cholangiography (ERCP), endoscopic ultrasonography (EUS) and positron emission tomography (PET). This study was undertaken to assess the comparative ability of USG and CT to diagnose the pancreatic lesions. Connor OJ, McWilliams S and Maher $\mathrm{MM}^{6}$ stated that the incidence of acute pancreatitis is approximately $5-70$ cases per 100,000 per year. Acute pancreatitis has a higher incidence in men than women, (generally between 40 and 50 years old with mean age of $42 \mathrm{yrs}$ ) but overall gallstone acute pancreatitis was most frequent in women. The clinical signs and symptoms most commonly include epigastric pain 
radiating to back. ${ }^{6} \mathrm{Kle} \mathrm{S}$, et al ${ }^{7}$ conducted a study to assess the clinical value of USG and CT in diagnosing and staging pancreatic carcinoma. The efficiency to detect the pancreatic carcinoma by routine ultrasound was 91.3 percent, while color-Doppler and 3-D techniques increased accuracy significantly upto 93.7 percent. Helical CT was shown to be the best modality of all, with accuracy reaching 94.4 percent $(\mathrm{p}>0.05)$. The routine ultrasound procedure achieved relatively low accuracy of $82.6 \%$ and color-Doppler imaging improved the accuracy upto $92.2 \%$ in assessment of vascular neoplastic infiltration. The study proved CT to be the most precise diagnostic tool in determining lymph node metastases; its accuracy reached $91.3 \%$, while all other modalities were below $90.0 \%{ }^{7}$

True epithelial cysts of the pancreas in adults are reported to be rare pathological lesions, although now being diagnosed more frequently due to the widespread use of USG, CECT, MRI and EUS, and their etiology still remains unknown. CECT of the abdomen revealed a hypodense structure measuring $4 \mathrm{~cm} \times 3.2 \mathrm{~cm} \times 2.3 \mathrm{~cm}$, located in the head of the pancreas, without any evidence of a solid component as reported in U Dalal case study. ${ }^{8}$ Histopathologically, the cystic lesion was a true pancreatic cyst. they concluded that CECT, USG and FNAC are sensitive for pre operative identification of the entity and for the differential diagnosis of other cystic pancreatic lesions and helps in management. ${ }^{8}$ Kulig J, et al ${ }^{9}$ stated that endoscopic ultrasound was the most accurate modality for local tumor staging (93.1\%), vascular infiltration (90\%), and lymph node assessment (87.5\%) than routine USG $(82.5 \%$ for local tumor staging, $67.5 \%$ for vascular infiltration, and $72.5 \%$ for lymph node assessment). The accuracy rate for CT and Doppler US were similar $(88.1 \%$ for local tumor staging, $82.5 \%$ for vascular infiltration and $80.0 \%$ for lymph node assessment). However, the advantage of endoscopic ultrasound over computed tomography and ultrasonography does not justify its routine use due to its high cost, low availability, and invasiveness. ${ }^{9}$

According to Lesniak RJ et al, ${ }^{10}$ ductal adenocarcinoma does not calcify and calcification may indicate underlying chronic calcific pancreatitis. In the present series also, calcification was seen in only 3 out of 10 cases of adenocarcinoma. CT could detect calcification in 11 cases compared to 9 cases on USG including one case of pancreatitis and another one of cystadenocarcinoma. Upadhyaya $\mathrm{V}$, et $\mathrm{al}^{11}$ used atleast two imaging modalities in each patient and findings were corroborated with the operative and/or histopathological findings. The overall diagnostic accuracy for detection of level of obstruction was maximum for ERCP/PTC (95.83\%), followed by MRCP (95.45\%), CT (85.71\%) and USG
(83.50\%). For assessing the cause, MRCP had the highest accuracy $(87.5 \%)$, followed by CT scan $(85.71 \%)$, USG (77\%) and ERCP/PTC (75\%). USG had traditionally been used as the initial screening procedure, however, although it was well suited to visualize the Common Hepatic Duct (CHD) and proximal CBD, one of its major limitations was in assessment of the distal CBD and pancreas, which were often obscured by overlying bowel gas in about $30-50 \%$ of the patients and obesity. In their study, USG missed many cases of CBD calculi. Other cases missed by USG were stricture, sclerosing cholangitis and cases of small mass lesions involving the head of pancreas.

Wong JC and Lu DS ${ }^{12}$ stated the sensitivity of CT for diagnosis of pancreatic adenocarcinoma (89\%-97\%) and its positive predictive value for predicting unresectability ( $89 \%$ $100 \%$ ) were high. The positive predictive value of CT for predicting resectability (45\%-79\%) was low because the diagnostic criteria for diagnosing vascular invasion by tumor favour specificity over sensitivity to avoid denying surgery to patients with potentially resectable tumor. Furthermore, the sensitivity of $\mathrm{CT}$ for small hepatic and peritoneal metastases was limited. Magnetic resonance imaging had not been shown to perform better than CT for the diagnosis and staging of pancreatic adenocarcinoma but could be helpful as an adjunct to CT, particularly for evaluation of small hepatic lesions that cannot be fully characterized by CT. Ultrasound has its limit for diagnosis and staging of patients with pancreatic adenocarcinoma. EUS was generally considered superior to $\mathrm{CT}$ for the diagnosis and local staging of pancreatic cancer, but was limited by availability and inability to assess for distant metastases. Takamitsu I, et al ${ }^{13}$ has evaluated pancreatic tumors preoperatively with positron emission tomography using F-18 fluorodeoxyglucose (FDG) and compared with CT and USG. In 33 of 35 patients, foci of pancreatic carcinomas (10-100 $\mathrm{mm}$ in diameter) were identified as an increase in FDG uptake, whereas CT and USG depicted the foci in 31 and 28 cases respectively. Among 11 benign lesions, nine showed no increased FDG uptake (specificity $=82 \%$ ). False positive findings were obtained in a case of chronic active pancreatitis and in a serous cystadenoma. The authors opined that FDG PET provided biochemical information and was accurate in identifying pancreatic carcinoma and could be a method of choice in imaging equivocal masses detected with anatomic imaging studies.

Berman L, et al ${ }^{14}$ presented a case of a patient with presumed intraductal papillary mucinous neoplasm (IPMN) who was ultimately found to have a serous cystadenoma in communication with the pancreatic duct. If EUS cannot be 
performed, resection was favored to avoid under treating a premalignant lesion. The most common were serous cystadenoma ( $32 \%$ to $39 \%$ ), intraductal papillary mucinous neoplasm (IPMN) (21\% to $33 \%)$, and mucinous cystic neoplasm (MCN) (10\% to 45\%). Solid-pseudopapillary tumors represent less than $10 \%$. Isolated retroperitoneal hydatid cyst is an exceptionally rare entity. Ultrasound abdomen revealed a lesion of size $7 \times 5 \mathrm{~cm}$ in retroperitoneal region in relation to the tail of pancreas. CECT of the abdomen revealed retroperitoneal lesion of size $7 \times 5 \mathrm{~cm}$ in relation to the tail of the pancreas with dilated small bowel loops. The rest of the organs were normal; hence, diagnosis of primary hydatid cyst of the pancreas was made radiologically. Ultrasound-guided aspiration was done again to rule out neoplastic nature of the lesion. Cytology revealed scolices compatible with hydatidosis. The histopathology of the cyst was compatible with hydatid cyst, but the attached capsule of pancreas overlying the cyst and tail was remarkably normal with no evidence of invasion. Hence, the final diagnosis of isolated primary retroperitoneal hydatid cyst of the pancreas was made, as other organs of the body did not reveal any hydatid cyst. ${ }^{15}$

Fine needle aspiration cytology together with imaging, had become a primary diagnostic modality for investigation of pancreatic mass lesions, both cystic and solid. Despite the advances in the imaging techniques, management options for patients were limited and a malignant diagnosis of solid lesions still carried a high mortality rate. This was based on the pre-operative distinction of non-mucinous and mucinous cysts in general, and benign and malignant cysts in particular. A cytological diagnosis could be obtained with minimally invasive techniques that utilize CT, US or EUS. Endoscopic Ultrasound guided FNA (EUS FNA) was evolving as the diagnostic method of choice due to its ability to more accurately stage the patient during a single procedure using EUS. ${ }^{16}$ For detecting cystic lesions, ultrasound with fineneedle aspiration had emerged as a prime modality investigations. Pancreatic cysts include inflammatory lesions, low-grade neoplasms, and malignant neoplasms. In the older literature, pseudocysts related to acute and chronic pancreatitis accounted for the majority of pancreatic cysts, but it was difficult to differentiate pancreatic cystic neoplasms from pseudocysts even with high-resolution modalities including computed tomography (CT) and magnetic resonance imaging (MRI) scans. ${ }^{17}$ With the development of USG and CT, the preoperative diagnosis of abdominal cystic disorder has become easy. USG can distinguish between solid and cystic masses and CT can determine extension and cystic content. ${ }^{18}$ The clinical data of 5 patients with MSAP were retrospectively analyzed. There were 5 female and 1 male.
USG and CT could detect macrocystic lesion of the pancreas; all the lesions showed a well-defined border, internal septations, enhanced cyst walls, with no intramural (mural) nodule and papillary projections; the wall was smooth and thin in 4 cases; irregular lobulation could be observed in 3 cases, 2 cases had pancreatic duct dilatation. The tumors were located in the pancreatic body and tail in 3 cases and pancreatic head in 2 cases. The size of the tumors ranged from $6.5 \mathrm{~cm}$ to $13.0 \mathrm{~cm}$ (mean, $8.8 \mathrm{~cm}$ ). ${ }^{19}$

Wakabayashi T, Kawaura Y, Satomura Y, et al ${ }^{20}$ reviewed 7 cases of chronic pancreatitis (CP) with focal narrowing of the main pancreatic duct (MPD), evidenced by ERCP, and swelling of one or two segments of the pancreas, evidenced by USG /CT. Stricture of the lower portion of the common bile duct (CBD) that caused obstructive jaundice was shown by ERCP in two cases. In all six patients, a dynamic study by CT or MRI homogeneously showed delayed enhancement of involved segments of the pancreas. The clinical, serologic, and histological findings as described above were comparable to those for $12 \mathrm{CP}$ patients with diffuse narrowing of the MPD, diagnosed during the same period. ${ }^{20}$ Vascular and perfusion images of contrast-enhanced ultrasound (CE-US) were used for the evaluation of tumor vascular-ity and parenchymal perfusion of the tumor, respectively. The hemodynamic of the tumor and the diagnostic capacity of CE-US were compared with those shown by computed tomography (CT). The endocrine tumors showed a heterogeneous hypervascular and hyperperfusion pattern. When tumors showing a hypovascular or hypoperfusion pattern on CE-US were diagnosed as carcinomas, 34 of the 39 carcinomas $(87 \%)$ fit this criterion, with a $95 \%$ confidence interval (CI) of $73 \%-96 \%$, whereas, on CT, 31 of the 39 were diagnosed as carcinoma; (sensitivity, $79 \%$ ). The sensitivity and accuracy of CT were inferior to those of CE-US. Results of comparison between the CE-US findings and the histological diagnosis were as follows. They concluded that the differences in vascularity of pancreatic carcinomas depicted by CE-US were associated well with differences in histology. ${ }^{21}$

Kamisawa T, Egawa N, Nakajima $\mathrm{H}$, et al $^{22}$ retrospectively examined findings of USG, CT, endoscopic retrograde cholangiopancreatography and angiography, in cases of pancreatic carcinoma, and compared histologically. USG showed an enlarged hypoechoic pancreas with sausage-like appearance and no lobulation in the contour of the pancreas. On computed tomography imaging, delayed enhancement of the swollen pancreatic parenchyma became evident. Bornman PC, Botha JF and Ramos JM et al ${ }^{23}$ studied that $\mathrm{CP}$ was a disease with significant clinical and pathological 
heterogeneity. The guidelines provide clear recommendations regarding the diagnostic modalities available, both imaging (which includes MRI and endoscopic ultrasound (EUS)) and pancreatic function tests. The section on medical management makes recommendations on the use of analgesics, enzyme replacement and other therapeutic options in the non-interventional management of the majority of patients with $\mathrm{CP}$. The section on interventional procedures identifies the indications and options available for the interventional management of both uncomplicated and complicated CP. The role of endoscopic and surgical modalities was defined, but it was in this context especially that the best available evidence, combined with the experience of the group, influenced the recommendations put forward. Owing to the lack of evidence and the complexity of the disease, it was recommended that, where possible, CP was managed in the context of a multidisciplinary team. ${ }^{23}$

Our study mounted the following points -

- CT has higher overall sensitivity and specificity for detecting and differentiating inflammatory and malignant lesions of pancreas as compared to USG.

- Sonography seems to be a good screening modality for evaluating patients with pancreatic lesions, because of its low-cost, ready availability, non-invasiveness and no radiation hazards to the patients. However, few cases remain non-diagnostic due to its technical limitations because of bowel gases, obesity and operatordependence.

- CT was far superior to USG in the evaluation of acute pancreatitis, detection peripancreatic inflammation, its extension into the retroperitoneal compartments and vascular involvement or metastasis

\section{Conclusion:}

$\mathrm{CT}$ and sonography are complementary imaging modalities in the evaluation of suspected pancreatic lesions. Thus in all the cases of pancreatic lesions, besides clinical examination; both these modalities (Sonography and CT scan) have their own role in detecting and differentiating lesions of pancreas and should be used in combination for accurate diagnosis and management. There is still a need to find a method which combines the accuracy of CT with similar availability and cost-effectiveness of USG.

\section{Conflict of Interest: None}

\section{References:}

1. Shanbhogue AKW, Fasih N, Surabhi VR, Doherty GP, Shanbhogue DKP, Sethi SK. A clinical and radiologic review of uncommon types and causes of pancreatitis. Radiographics. 2009;29:1003-26.

2. Clarke DL, Thomson SR, Madiba TE, Sanyika C. Preoperative imaging of pancreatic cancer: a managementoriented approach. J Am Coll Surg. 2003;196(1):119-29.

3. Kalra MK, Maher MM, Mueller PR, Saini S. State-of-theart imaging of pancreatic neoplasms. $\mathrm{Br} J$ Radiol. 2003;76(912):857-65.

4. Hilendarov AD, Velkova KG. Ultrasound tomography in diagnosing cystic pancreatic neoplasms. Folia Med (Plovdiv). 2011;53(1):34-9.

5. Minniti S, Bruno C, Biasiutti C, Tonel D, Falzone A, Falconi $\mathrm{M}$, et al. Sonography versus helical CT in identification and staging of pancreatic ductal adenocarcinoma. J Clin Ultrasound. 2003;31(4):175-82.

6. Connor OJ, McWilliams S, Maher MM. Imaging of Acute Pancreatitis. AJR. 2011;197:221-25.

7. KBek S, Kulig J, Popiela T, KoBodziejczyk P, SzybiDski P, Popiela TJ, et al. The value of modern ultrasonographic techniques and computed tomography in detecting and staging of pancreatic carcinoma. Acta Chir Belg. 2004;104(6):659-67.

8. Dalal U, Singal R, Dalal AK, Kumar Y, Gupta S, Mendiratta $\mathrm{P}$, et al. Enucleation of the solitary epithelial cyst of pancreatic head in an adult: a case report and review of the literature. Niger J Clin Pract. 2012;15(2):228-30.

9. Kulig J, Popiela T, Zajac A, KBek S, KoBodziejczyk P. The value of imaging techniques in the staging of pancreatic cancer. Surg Endosc. 2005;19(3):361-5.

10. Robert J Lesniak, Mark D Howen Walter, Andrew J Taylor. Spectrum of causes of pancreatic calcifications. AJR. 2002;178:79-86.

11. Upadhyaya V, Upadhyaya DN, Ansari MA, Shukla VK. Comparative assessment of imaging modalities in biliary obstruction. Indian J Radiol Imaging. 2006;16:577-82.

12. Wong JC, Lu DS. Staging of pancreatic adenocarcinoma by imaging studies. Clin Gastroenterol Hepatol. 2008;6(12):1301-8.

13. Takamitsu I, Shigeki M, Kazuo S, Yoshiteru K, Kenya C, Masato F. Spontaneously Ruptured Giant Splenic Cyst with Elevated Serum Levels of CA 19-9, CA 125 and Carcinoembryonic Antigen. Case Rep Gastroenterol. 2010;4(2):191-97.

14. Berman L, Mitchell KA, Israel G, Salem RR. Serous Cystadenoma in Communication with the Pancreatic Duct: An Unusual Radiologic and Pathologic Entity. Journal of Clinical Gastroenterology. 2010;44(6):133-35.

15. Dalal U, Dalal AK, Singal R, Naredi B, Gupta S. Primary hydatid cyst masquerading as pseudocyst of 
the pancreas with concomitant small gut obstruction-an unusual presentation. Kaohsiung J Med Sci. 2011;27(1): $32-5$.

16. Kocjan G. Fine needle aspiration cytology of the pancreas: a guide to the diagnostic approach. Coll Antropol. 2010;34(2):749-56.

17. Friedel DM, Abraham B, Georgiou N, Stavropoulos SN, Grendell JH, Katz DS. Pancreatic cystic neoplasms. South Med J. 2010;103(1):51-7.

18. Singal R, Gupta S, Singh B. Successful enucleation of retroperitoneal cyst. J Ayub Med Coll Abbottabad. 2012;24:212-4.

19. Wang YL, Guo KJ, Zhao MF, Song SW, Xu YH, Ma G. Macrocystic serous adenoma of the pancreas: a report of 5 cases. Zhonghua Wai Ke Za Zhi. 2010;48(18):1405-8.

20. Wakabayashi T, Kawaura Y, Satomura Y, Fujii T, Motoo Y, Okai T, et al. Clinical Study of Chronic Pancreatitis with
Focal Irregular Narrowing of the Main Pancreatic Duct and Mass Formation: Comparison with Chronic Pancreatitis Showing Diffuse Irregular Narrowing of the Main Pancreatic Duct. Pancreas. 2002;25(3):283-9.

21. Sofuni A, Iijima H, Moriyasu F, Nakayama D, Shimizu M, Nakamura K, et al. Differential diagnosis of pancreatic tumors using ultrasound contrast imaging. J Gastroenterol. 2005;40(5):518-25.

22. Kamisawa T, Egawa N, Nakajima H, Tsuruta K, Okamoto A, Kamata N, et al. Comparison of radiological and histological findings in autoimmune pancreatitis. Hepatogastroenterology. 2006;53(72):953-6.

23. Bornman PC, Botha JF, Ramos JM, Smith MD, Van der Merwe S, Watermeyer GA, et al. Guideline for the diagnosis and treatment of chronic pancreatitis. S Afr Med J. 2010;100:845-60. 